\title{
Isentropic fitting of Anosov automorphisms
}

\author{
DAVID FRIED \\ Department of Mathematics, University of California, Santa Cruz, California 95064
}

(Received 12 March 1981 and revised 2 April 1982)

Abstract. In response to a question of Newhouse, we show that in many cases an Anosov automorphism can be uniformly approximated by a Smale diffeomorphism of the same topological entropy. We conjecture this can be done whenever the automorphism is sufficiently hyperbolic.

A three-dimensional example is given that suggests this approximation is not always possible.

\section{Introduction}

We will study and partly solve a problem of S. Newhouse concerning the geometric realization of the symbolic dynamics of an Anosov automorphism. Our results give many non-trivial new examples of isotopy classes that contain simplest representatives in the sense of Shub [10].

The two best understood classes of structurally stable diffeomorphisms are the Anosov automorphisms and the Smale diffeomorphisms, defined as follows. Let $\boldsymbol{N}$ be a simply connected nilpotent Lie group, $\Gamma$ a discrete cocompact subgroup and $\alpha$ a Lie group automorphism of $N$ that preserves $\Gamma$. The induced map $\bar{\alpha}: N / \Gamma \rightleftharpoons$ is an automorphism of the nilmanifold $N / \Gamma$. If the differential $D \alpha(e)$ of $\alpha$ at the identity $e \in N$ is hyperbolic we call $\bar{\alpha}$ Anosov. A Smale diffeomorphism is a diffeomorphism $f$ of a closed manifold whose chain-recurrent set $R(f)$ is hyperbolic and 0-dimensional. Amongst structurally stable diffeomorphisms these lie at opposite extremes in that $R(\bar{\alpha})$ has codimension 0 and $R(f)$ has dimension 0 .

By well-known results of Sinai and Bowen $([12],[1])$ each of these systems is described by symbolic dynamics. Sinai showed $\bar{\alpha}$ is a factor of a subshift of finite type of the same topological entropy (we will call systems of equal entropy isentropic). Bowen showed that $f \mid R(f)$ is conjugate to a subshift of finite type. Newhouse asked for a direct connection between these two classes of dynamical systems. Question: Can an Anosov automorphism $\bar{\alpha}$ be deformed by a $C^{0}$-small isotopy to an isentropic Smale diffeomorphism $f$ ? If it can we say that $\bar{\alpha}$ has isentropic Smale approximations (ISA's). By a result of Franks [4], there is a semiconjugacy from $f$ onto $\bar{\alpha}$, so that $R(f)$ gives symbolic dynamics for $\bar{\alpha}$. Shub used this semiconjugacy to show that an ISA for $\bar{\alpha}$ is a simplest diffeomorphism, meaning that it is Smale and its entropy is as small as possible in its isotopy class [10]. 
Some motivation for this problem arises from the case $N / \Gamma=\mathbb{R}^{2} / \mathbb{Z}^{2}$. Then one may apply Smale's DA construction twice to $\bar{\alpha}$ to produce an ISA $f$. So what is needed is an alternative to the DA construction in higher dimensions.

When $\bar{\alpha}$ had no real eigenvalues it was previously unknown whether there were any isentropic maps isotopic to $\bar{\alpha}$ besides other Anosov diffeomorphisms. Moreover there are ergodic (non-hyperbolic) toral automorphisms that do not have ISA's [6]. But we will produce an Anosov automorphism without real eigenvalues that does admit ISA's. We use the fitted diffeomorphisms of Shub \& Sullivan [11]. This and our other examples are evidence for the conjecture of Shub that the lowest entropy amongst structurally stable diffeomorphisms in an isotopy class arises for a fitted diffeomorphism [10].

These fitted diffeomorphisms are Smale diffeomorphisms that preserve the skeleta $\mathscr{H}_{i}$ of some handle decomposition $\mathscr{H}$ (for full definitions and proofs, see [5], [11]). Given some ordering and orientations for the $i$-handles, a fitted diffeomorphism determines two matrices $A_{i}(f)$ and $G_{i}(f)$ that discretize the topology and dynamics of $f$ respectively. Here $G_{i}(f)$ is the geometric intersection matrix: its $(j, k)$ 'th entry measures how many times the $j$ 'th transverse disk meets the image under $f$ of the $k$ 'th core disk. $\boldsymbol{A}_{i}(f)$ is the algebraic intersection matrix that counts these intersections with weights +1 or -1 according to the orientations. Writing $\left|A_{i}(f)\right|$ for the non-negative matrix whose entries are the absolute values of those of $A_{i}(f)$, we have the basic inequality (holding entry by entry) $\left|A_{i}(f)\right| \leq G_{i}(f)[11]$.

It is important for our purposes not only to construct fitted $f$ 's but to compute the topological entropy $h(f)$. For a linear endomorphism $\xi$ of a finite dimensional real vector space (or a matrix) we let

$$
L(\xi)=\log (\sup |\lambda|)
$$

where $\lambda$ varies over the eigenvalues of $\xi$ by the logarithmic spectral radius of $\xi$. For a fitted $f$,

$$
h(f)=\sup _{i} L\left(G_{i}(f)\right)
$$

[11].

A somewhat similar formula for Anosov automorphisms is due to Bowen. If

$$
e=N^{0} \subset N^{1} \subset \cdots \subset N^{k}=N,
$$

with $N^{i+1} / N^{i}$ the centre of $N / N^{i}$ and $N^{i+1} \neq N^{i}$ for all $i$, is the ascending central series for $N$ then $N / N^{i} \Gamma$ is a nilmanifold that fibres over the nilmanifold $N / N^{i+1} \Gamma$ with fibre a torus $N^{i+1} \Gamma / N^{i} \Gamma$. The automorphism $\alpha$ induces linear transformations

$$
\alpha_{i}: N^{i+1} / N^{i} \rightleftharpoons
$$

Then $h(\bar{\alpha})=\sum_{i} L\left(\alpha_{i}\right)[2]$.

The contents of this paper are as follows. In $\S 1$ we simplify the Newhouse problem and reduce it to a special case of Shub's conjecture. Namely we show that it suffices to find an isentropic Smale diffeomorphism in the isotopy class of each 
irreducible Anosov toral automorphism. Also we show that the characteristic polynomial (not the integral conjugacy class) of $\alpha$ is all that really matters here.

In $\S 2$ we set up our tools for the isentropic fitting of toral automorphisms. We use these in $\S 2$ to redo the dimension two case and in $\S 3$ for our new results in dimensions three and four. Finally we give in $\S 4$ an Anosov automorphism of $T^{3}$ for which the methods of $\S 2$ cannot give an isentropic Smale map. These examples lead us to conjecture that Newhouse's question has an affirmative answer for sufficiently hyperbolic automorphisms, as follows.

CONJECTURE. There is a number $k(d)$ depending only on the dimension $d$ of $N / \Gamma$. such that an Anosov automorphism $\bar{\alpha}: N / \Gamma \rightleftharpoons$ has ISA's whenever every eigenvalue $\lambda$ of $D \alpha(e)$ satisfies $|\log | \lambda \mid>k(d)$.

This is shown to hold for $d=3$ provided stable and unstable orientations are preserved (theorem 2).

We remark that Newhouse's question is interesting for other Axiom $\boldsymbol{A}$ systems as well. Since transitive Anosov diffeomorphisms have the largest possible nonwandering sets, this would seem to be the most difficult case. As all known Anosov diffeomorphisms are finitely covered by Anosov diffeomorphisms of nilmanifolds and the latter are topologically conjugate to Anosov automorphisms [8] the question as posed above is of special importance.

We thank Sheldon Newhouse for prompting this research.

\section{Some simplifications}

In this section we show that Newhouse's question reduces to the case of minimal toral automorphisms. We also show that if two minimal toral automorphisms have the same characteristic polynomial then one admits ISA's if and only if the other does. Both arguments rely on constructing fibre preserving isotopies of the total space of a fibration.

Let $G$ be a Lie group and $\beta \in$ Aut $(G)$. Recall that if $F, F^{\prime}$ admit transitive $G$-actions then a diffeomorphism $h: F \rightarrow F^{\prime}$ is an affine automorphism with linear part $\beta$ if for all $x \in F$ and $g \in G$,

$$
h(g x)=\beta(g) h(x)
$$

We study families of affine automorphisms of tori in the next lemma.

LEMMA 1. Suppose $\pi: E \rightarrow B$ is a smooth principal $T^{n}$ bundle over a closed manifold $B$. Let $f: E \rightleftharpoons$ be a diffeomorphism such that

$$
f(g x)=\beta(g) f(x)
$$

for some $\beta \in$ Aut $\left(T^{n}\right)$ and all $x \in E, g \in T^{n}$. Let $\bar{f}: B \rightleftharpoons$ be the induced diffeomorphism.

If $\beta$ is isotopic to a Smale diffeomorphism $\gamma$ and if $\bar{f}$ is Smale then there is a $C^{0}$-small isotopy $f_{\mathrm{s}}$ from $f_{0}=f$ to a Smale $f_{1}$ such that each $f_{\mathrm{s}}$ induces $\bar{f}$ and

$$
h\left(f_{1}\right)=h(\gamma)+h(\bar{f}) .
$$


Proof. As $R(\bar{f}) \subset B$ is 0-dimensional, we can find a compact submanifold $V \subset B$ of codimension 0 such that

$$
R(\bar{f}) \subset \operatorname{int}(V)
$$

and each component of $V$ is small. Thus the given bundle is trivial over $V$ and we identify $\pi^{-1}(V)$ with $V \times T^{n}$.

Let $W \subset V \cap \bar{f}^{-1} V$ be a neighbourhood of $R(\bar{f})$. Then on $W \times T^{n}$ we have

$$
f(w, g)=(\bar{f}(w), \beta(g)+\phi(w))
$$

for some smooth $\phi: W \rightarrow T^{n}$.

Now choose a large positive integer $q$. Restricting $W$ if necessary, we deform $\phi$ to $\psi: W \rightarrow T^{n}$ such that $q \psi=0$ by a homotopy of size proportional to $1 / q$. We identify $q$ with the covering map $q: T^{n} \circlearrowright$ that sends $g$ to $q \mathrm{~g}$. We lift our isotopy from $\beta$ to $\gamma$ under the covering $q$ to an isotopy from $\beta$ (note that $\beta$ commutes with $q$ ) to some lift $\gamma_{q}$ of $\gamma$. This new isotopy also has size proportional to $1 / q$.

Combining the new isotopy of $\beta$ with the homotopy of $\phi$ gives a small isotopy from $f \mid \pi^{-1} W$ to $f_{1}$ where

$$
f_{1}(w, g)=\left(\bar{f}(w), \gamma_{q}(g)+\psi(w)\right) .
$$

As all the maps in this isotopy induce $\bar{f}$ over $W$, we can extend $f_{\text {s }}$ to a small isotopy of $E$ from $f$ to $f_{1}$ keeping this property.

It follows that

$$
\pi\left(R\left(f_{1}\right)\right) \subset \boldsymbol{R}(\bar{f}) \subset \boldsymbol{W} .
$$

Also

$$
\left(\bar{f}(w), q \gamma_{q}(g)+\psi(w)\right)=(\bar{f}(w), \gamma(q g)+q \psi(w))=(\bar{f}(w), \gamma(q g)) .
$$

Thus under the finite covering

$$
\hat{q}=\mathrm{id} \times q: W \times T^{n} P, f_{1}
$$

is the lift of $\bar{f} \times \gamma$. As the latter map is Smale, it follows easily that $f_{1}$ is Smale. Finally

$$
\begin{aligned}
h\left(f_{1}\right) & =h\left(f_{1} \mid R\left(f_{1}\right)\right) & & \text { by [3] } \\
& =h(\bar{f} \times \gamma \mid R(f) \times R(\gamma)) & & \text { as } \hat{q} \text { is a finite cover } \\
& =h(\bar{f} \mid R(\bar{f}))+h(\gamma \mid R(\gamma)) & & \text { by [3] } \\
& =h(\bar{f})+h(\gamma) & & \text { by [3] again. }
\end{aligned}
$$

Now suppose $\bar{\alpha}: N / \Gamma \supset$ is an Anosov automorphism. For each $i$ we choose a filtration of maximal length of the torus $N^{i+1} \Gamma / N^{i} \Gamma$ by invariant tori. This gives an $\alpha$-invariant filtration

$$
\Gamma=G_{0} \Gamma \subsetneq G_{1} \Gamma \subsetneq \cdots \varsubsetneqq G_{m} \Gamma=N
$$

with $G_{i}$ simply connected for all $i$ such that each quotient torus $G_{i} \Gamma / G_{i-1} \Gamma$ has an induced Anosov automorphism $\bar{\alpha}_{i}$. We call $\bar{\alpha}_{i}$ the irreducible parts of $\bar{\alpha}$ (although they depend on the choice of filtration as well). Then we have

THEOREM 1. Each irreducible part is an irreducible Anosov toral automorphism. If each irreducible part can be isotoped to an isentropic Smale diffeomorphism then $\bar{\alpha}$ admits ISA's. 
Proof. The first statement is clear, as the maximality of our filtration implies that the linear maps

$$
\alpha_{i}: G_{i} / G_{i-1} \rightleftharpoons
$$

have irreducible characteristic polynomial over the rationals.

The second statement is shown by induction on the length of the filtration. If the Anosov automorphism

$$
\bar{\beta}_{i}: N / G_{i} \Gamma \rightleftharpoons
$$

induced by $\alpha$ admits ISA's, we show that $\bar{\beta}_{i-1}$ also does. As $\bar{\beta}_{m-1}$ is an irreducible part and $\bar{\beta}_{0}=\bar{\alpha}$, this will prove the theorem.

We regard $E^{\prime}=N / \Gamma$ as a bundle over $B=N / G_{i}$ with fibre $F^{\prime}=G_{i} \Gamma / \Gamma$, all three spaces being nilmanifolds. Let $\gamma$ be the restriction of $\alpha$ to $G_{i}$. Then $\bar{\kappa}$ is a diffeomorphism of $E^{\prime}$ that satisfies

$$
\bar{\alpha}(g x)=\gamma(g) \bar{\alpha}(x), \quad x \in E^{\prime}, g \in G_{i} .
$$

Hence $\bar{\alpha}$ preserves fibres, acts on each fibre by an affine automorphism with linear part $\gamma$ and induces $\vec{\beta}_{i}$ on $B$.

Let $\bar{f}: B \rightleftharpoons$ be an ISA for $\bar{\beta}_{i}$, given by our inductive hypothesis. We can lift the small isotopy from $\bar{\beta}_{i}$ to $\bar{f}$ to an isotopy $F_{t}$ from $\bar{\alpha}=F_{0}$ to some $F_{1}$ so that

$$
F_{t}(g x)=\gamma(g) F_{t}(x)
$$

for all $t \in[0,1], x \in E, g \in G_{i}$.

It follows that $F_{t}$ induces an isotopy $\hat{F}_{t}$ on $N / G_{i-1} \Gamma$. Denoting the latter by $E$, it is a principal torus bundle over $B$. If $f=\hat{F}_{1}$ then $f: E \circlearrowright$ induces $\bar{f}: B \rightleftharpoons$ and satisfies the conditions of the lemma. So we can make a small isotopy from $f$ to a Smale diffeomorphism $f_{1}$ with

$$
h\left(f_{1}\right)=h(\bar{f})+h(\delta)
$$

where $\delta$ is an ISA for the irreducible part $\bar{\alpha}_{i}$. Thus

$$
h\left(f_{1}\right)=h\left(\bar{\beta}_{i}\right)+h\left(\bar{\alpha}_{i}\right)=h\left(\bar{\beta}_{i-1}\right),
$$

so $f_{1}$ is an ISA for $\overline{\boldsymbol{\beta}}_{i-1}$.

We now show

LEMMA 2. Suppose $\beta, \gamma \in \mathrm{Gl}(n, \mathbb{Z})$ both have characteristic polynomial $p(x)$ and that $p(x)$ is irreducible over the rationals. If $\bar{\beta}$ can be isotoped to an isentropic Smale diffeomorphism then so can $\bar{\gamma}$.

Proof. As $p(x)$ is irreducible both $\beta$ and $\gamma$ are similar over the rationals to the companion matrix of $p(x)$. So we may regard $\beta$ and $\gamma$ as being restrictions to invariant lattices $L_{\beta}, L_{\gamma}$ of rational points in an $n$-dimensional real vector space $V$ of a linear map $P: V P$. The maps $\bar{\beta}, \bar{\gamma}$ are respectively the action induced by $P$ on $V / L_{\beta}, V / L_{\gamma}$. Choose $q$ a positive integer so that $q L_{\gamma} \subset L_{\beta}$. Then multiplication by $q$ induces a covering $V / L_{\gamma} \rightarrow V / L_{\beta}$. We lift the isotopy from $\bar{\beta}$ to $f_{\beta}$ (an isentropic 
Smale diffeomorphism) to an isotopy from $\bar{\gamma}$ to some Smale diffeomorphism $f_{\gamma}$. We have

$$
h\left(f_{\gamma}\right)=h\left(f_{\beta}\right)=h(\bar{\beta})=L(\beta)=L(\gamma)=h(\bar{\gamma}),
$$

so $f_{\gamma}$ and $\bar{\gamma}$ are isentropic.

In summary, we have shown that in order to answer Newhouse's question it suffices to find for each monic irreducible polynomial $p(x) \in \mathbb{Z}[x]$ with $p(0)= \pm 1$ and no roots on $S^{1}$ a linear toral automorphism $\bar{\alpha}$ with $p(x)$ as characteristic polynomial and an isentropic Smale diffeomorphism isotopic to $\bar{\alpha}$. The same holds for our conjecture since the eigenvalues of $D \alpha(e)$ are just the eigenvaiues of the irreducible parts of $\bar{\alpha}$.

\section{Fitted isentropic maps}

To find isentropic approximations to maps on tori, we will construct diffeomorphisms that are fitted relative to a standard handle decomposition $\mathscr{H}$ of $T^{n}$, defined below.

There is a cell complex structure on $\mathbb{R}$ with one 0 -cell $\{m\}$ and one 1 -cell $[m, m+1]$ for each $m \in \mathbb{Z}$. The Cartesian $n$th power defines a cell complex on $\mathbb{R}^{n}$ that is invariant under $\mathbb{Z}^{n}$. The associated cell complex on $T^{n}=\mathbb{R}^{n} / \mathbb{Z}^{n}$ has one $p$-cell $c(I)$ for each $p$ element subset $I \subset\{1, \ldots, n\}$. If $J$ is the complement of $I$ then

$$
c(I)=\left\{\left(v_{1}, \ldots, v_{n}\right) \in \mathbb{R}^{n} \mid v_{j} \in \mathbb{Z} \text { for all } j \in J\right\} / \mathbb{Z}^{n} .
$$

Let $U_{p}$ be the $\varepsilon(p)$ neighbourhood of the $p$-skeleton where the $\varepsilon(p)$ decrease rapidly, $p=0,1, \ldots, n$. Then

$$
U_{p}-\operatorname{int}\left(\bigcup_{q<p} U_{q}\right)
$$

is the union of $\left(\begin{array}{l}n \\ p\end{array}\right)$ disjoint $p$-handles, defining the standard handle decomposition $\mathscr{H}$ of $T^{n}$.

We assume some familiarity with the notion of fitted diffeomorphism as summarized in the introduction. We have the following criterion for a fitting of a toral automorphism relative to $\mathscr{H}$ to be isentropic.

LeMMA 3. Suppose that $f: T^{n} \rightleftharpoons$ is fitted for $\mathscr{H}$ and isotopic to $\bar{\alpha}: T^{n} \rightleftharpoons$. Let $u$ be the number of eigenvalues of modulus $>1$ for $\alpha$. Then $f$ and $\bar{\alpha}$ are isentropic

$$
\Leftrightarrow L\left(G_{p}(f)\right) \leq L\left(\Lambda^{u} \alpha\right)
$$

for all $p=1, \ldots, n-1$.

If this holds then $L\left(G_{u}(f)\right)=L\left(A_{u}(f)\right)$. If also $\Lambda^{u} \alpha$ is irreducible then $G_{u}(f)$ and $\pm A_{u}(f)$ have the same characteristic polynomial and $G_{u}(f)=\left|A_{u}(f)\right|$.

Proof. First we observe that $A_{p}(f)$ represents the action of $f$ on $p$ 'th homology. This holds because $\mathscr{H}$ has unly as many $p$-handles as the rank of $H_{p}\left(T^{n} ; \mathbb{Z}\right)$.

Secondly, the cup product structure of $H^{*}\left(T^{n} ; \mathbb{Z}\right)$ gives

$$
H^{p}\left(T^{n} ; \mathbb{Z}\right)=\Lambda^{p} H^{1}\left(T^{n} ; \mathbb{Z}\right) .
$$

Dualizing, $A_{p}(f)$ is a matrix representation for $\Lambda^{p} \alpha$ and so

$$
L\left(A_{p}(f)\right)=L\left(\Lambda^{p} \alpha\right) .
$$


As $\left|A_{p}(f)\right| \leqslant G_{p}(f)$, we have

$$
L\left(\Lambda^{p} \alpha\right) \leq L\left(G_{p}(f)\right)
$$

for all $p$. Now we use the formulae for $h(\bar{\alpha})$ and $h(f)$ from the introduction to obtain our first two conclusions.

Let $\lambda_{1}, \ldots, \lambda_{u}$ be the eigenvalues of $\alpha$ of modulus $>1$. Then $\lambda_{1} \cdots \lambda_{u}=\lambda$ is the eigenvalue of $\Lambda^{u} \alpha$ of largest modulus. Thus

$$
L\left(G_{u}(f)\right)=\log |\lambda|,
$$

so Perron-Frobenius theory implies $|\lambda|$ is an eigenvalue of $G_{u}(f)$. But $\lambda \in \mathbb{R}$ since unstable eigenvalues come in conjugate pairs. Thus $\pm A_{u}(f)$ and $G_{u}(f)$ have a common eigenvalue. If $\Lambda^{u} \alpha$ is irreducible, then these matrices must have the same characteristic polynomial.

Finally since $\Lambda^{u} \alpha$ is irreducible over the rationals, $\left|A_{u}(f)\right|$ is irreducible as a non-negative matrix. Since

$$
L\left(G_{u}(f)\right)=L\left(\left|A_{u}(f)\right|\right)
$$

we must have

$$
G_{u}(f)=\left|A_{u}(f)\right| .
$$

The preceding lemma shows that an isentropic fitting relative to the standard handle decomposition is severely constrained in the unstable dimension. The proof also shows that $A_{\beta}(f)$ is the exterior power $\Lambda^{p} A_{1}(f)$ if one properly orders and orients the $p$-handles of $\mathscr{H}$.

We will construct fitted diffeomorphisms by composing the simple ones of the following lemma.

LEMMA 4. Let $\gamma$ be an elementary matrix with diagonal entries 1 and one off-diagonal entry \pm 1 other than 0 . Then $\bar{\gamma}$ can be fitted for $\mathscr{H}$ with $\left|A_{p}\right|=G_{p}$.

The same holds for $\gamma$ a signed permutation matrix.

Proof. In the first case, we can reorder and reorient our basis so that

$$
\gamma=\delta \times I, \delta=\left(\begin{array}{ll}
1 & 1 \\
0 & 1
\end{array}\right)
$$

Fit $\bar{\delta}$, as in figure 1 , by spreading the shearing in a non-linear way. Crossing with the identity map and perturbing gives the desired fitting for $\bar{\gamma}$.

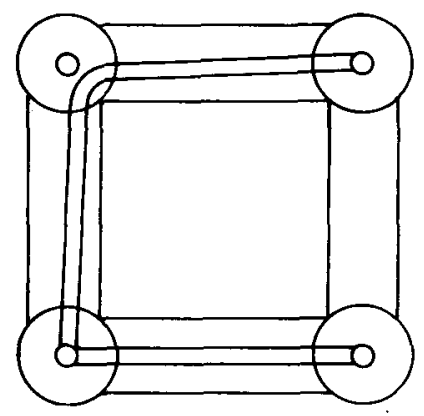

FIGURE 1. Fitting $\bar{\delta}$. 
In the second case, we can approximate $\bar{\gamma}$ by a fitted map with $A_{1}=\gamma$.

In each case, the geometric intersection matrices only have entries 0 and 1 , so $G_{p}=\left|A_{p}\right|$ for all $p$.

To illustrate the use of these lemmas, let $n=2$. Let

$$
\gamma_{1}=\left(\begin{array}{ll}
1 & 1 \\
0 & 1
\end{array}\right), \quad \gamma_{2}=\left(\begin{array}{ll}
1 & 0 \\
1 & 1
\end{array}\right), \quad \gamma_{3}=\left(\begin{array}{ll}
0 & 1 \\
1 & 0
\end{array}\right), \quad \text { and } \quad \gamma_{4}=\left(\begin{array}{rr}
-1 & 0 \\
0 & -1
\end{array}\right)
$$

We fit each $\gamma_{i}$ by $f_{i}$ so that $G_{1}\left(f_{i}\right)= \pm \gamma_{i}$, as in lemma 4 .

Let $a$ be an integer and $b= \pm 1$ and suppose that

$$
p(x)=x^{2}-a x+b
$$

has no roots on $S^{1}$. If $b=+1$ then $|a| \geq 3$ and if $b=-1$ then $a \neq 0$. Let $\sigma= \pm 1$ be the sign of $a$. If $b=-1$ we let $\alpha=\sigma \gamma_{3} \gamma_{1}^{|a|}$ and if $b=+1$ we let $\alpha=\sigma \gamma_{2} \gamma_{1}^{|a|-2}$. It is easy to see that the characteristic polynomial of $\alpha$ is $p(x)$.

We define a fitted diffeomorphism $f$ isotopic to $\bar{\alpha}$ by replacing $\gamma_{i}$ by $f_{i}$ throughout. As geometric intersection matrices multiply under composition, $G_{1}(f)= \pm \alpha$ so that $f$ and $\bar{\alpha}$ are isentropic (lemma 3 ). By $\S 1$, all Anosov automorphisms of $T^{2}$ have ISA's. So we have recovered, without use of the DA construction, the results for $T^{2}$ mentioned in the introduction.

\section{Results in dimensions three and four}

We now present our examples on $T^{3}$ and $T^{4}$.

THEOREM 2. There is a constant $k>0$ so that any automorphism $\bar{\alpha}$ of $T^{3}$ that preserves the orientations of its stable and unstable manifolds and that has no eigenvalues in the annulus

$$
e^{-k}<|z|<e^{k}
$$

admits ISA's.

Proof. By $\S 1$, it will suffice to show that some Anosov automorphism $\bar{\beta}$ with the same characteristic polynomial

$$
p(x)=x^{3}-A x^{2}+B x-1
$$

admits an isentropic fitting $f$ relative to the standard handle decomposition of $T^{3}$. As then $f^{-1}$ is fitted for the dual handle decomposition, we may suppose by passing to inverses that $u=1$.

Thus $p(x)$ has one root $\lambda>0$ outside the unit disk and two roots $\mu, \nu$ inside. We have $\lambda=e^{h} \gg 1$ and $|\mu|,|\nu| \ll 1$. As $A=\lambda+\mu+\nu$ and $B=\lambda \mu+\lambda \nu+\mu \nu$, we have $A \gg 1$ and $|B / A| \ll 1$.

First suppose $B \leq 0$. Then we take

$$
\beta=\left(\begin{array}{ccc}
0 & 1 & -B \\
0 & 0 & 1 \\
1 & 0 & A
\end{array}\right)=\left(\begin{array}{ccc}
0 & 1 & 0 \\
0 & 0 & 1 \\
1 & 0 & 0
\end{array}\right)\left(\begin{array}{ccc}
1 & 0 & 1 \\
0 & 1 & 0 \\
0 & 0 & 1
\end{array}\right)^{A}\left(\begin{array}{ccc}
1 & 0 & 0 \\
0 & 1 & 1 \\
0 & 0 & 1
\end{array}\right)^{-B}
$$

It is easy to see that $\beta$ has $p(x)$ as characteristic polynomial. Fit each factor using lemma 4 and compose to obtain a fitted diffeomorphism $f$ isotopic to $\bar{\beta}$ with 
$G_{1}(f)=\beta$. Taking inverse transposes of the factors, then absolute values and multiplying gives $G_{2}(f)$. One can check that the characteristic polynomial $q(x)$ of $G_{2}$ is $x^{3}+B x^{2}-A x-1$. We have $q(1)<0$ so $q$ has exactly one root $\xi \in(1, \infty)$. As

$$
q(\lambda)=(A+B)\left(\lambda^{2}-\lambda\right)>0, \lambda>\xi
$$

Thus $h(f)=\log \lambda=h(\bar{\beta})$ so we are done in this case.

Thus we may suppose $B>0$. Let $v=A-1, C=v-B \geq 0$ and

$$
\beta=\left(\begin{array}{lll}
0 & 0 & 1 \\
1 & 0 & 0 \\
0 & 1 & 0
\end{array}\right)\left(\begin{array}{lll}
1 & 1 & 0 \\
0 & 1 & 0 \\
0 & 0 & 1
\end{array}\right)^{C}\left(\begin{array}{lll}
1 & 0 & 0 \\
0 & 1 & 1 \\
0 & 0 & 1
\end{array}\right)\left(\begin{array}{lll}
1 & 1 & 0 \\
0 & 1 & 0 \\
0 & 0 & 1
\end{array}\right)^{B}=\left(\begin{array}{lll}
0 & 0 & 1 \\
1 & v & C \\
0 & 1 & 1
\end{array}\right)
$$

this time. Using lemma 4 on each factor, we produce a fitted diffeomorphism $f$ in the isotopy class of $\bar{\beta}$. As above, one shows that the characteristic polynomial of $G_{2}(f)$ is

$$
\tilde{q}(x)=x^{3}-B x^{2}-A x-1,
$$

and that the roots of $\tilde{q}(x)$ have modulus $<\lambda$. As $G_{1}(f)=\beta$ has $p(x)$ as characteristic polynomial, $f$ and $\bar{\beta}$ are isentropic.

We obtain the following partial solution to Newhouse's problem in dimension three.

Corollary. For any Anosov diffeomorphism $A$ in dimension three, some iterate of $A$ admits ISA's.

Proof. By [9] $A$ is conjugate to an automorphism of $T^{3}$. The preceding theorem then applies to some iterate.

We now give an Anosov automorphism of $T^{4}$ with no real eigenvalues that has an isentropic fitting and so, by $\S 1$, admits ISA's.

Let $v_{1}, \ldots, v_{4}$ be the standard basis for $\mathbb{R}^{4}$. Let $\pi$ be the signed permutation matrix sending

$$
v_{1} \mapsto v_{2} \mapsto v_{3} \mapsto-v_{4}
$$

Let $\beta$ fix $v_{2}, v_{3}, v_{4}$ and let

$$
\beta\left(v_{1}\right)=v_{1}+v_{2}-v_{4}
$$

Then let $\alpha=\beta^{d} \pi$ be the companion matrix of

$$
p(x)=x^{4}-d x^{3}+d x-1 \text {. }
$$

For the basis $v_{i} \wedge v_{i}, i<j$, of $\Lambda^{2} \mathbb{R}^{4}$ the matrices $\Lambda^{2} \pi, \Lambda^{2} \beta$ are non-negative. Fitting $\bar{\pi}$ and $\bar{\beta}$ in the obvious way defines a fitting $f$ for $\bar{\alpha}$.

Computing $G_{1}(f)$ and $G_{3}(f)$ we find that they have the same characteristic polynomial

$$
q(t)=t^{4}-d t^{3}-d t-1=\left(t^{2}+1\right)\left(t^{2}-d t-1\right) .
$$

As $G_{2}(f)=\Lambda^{2}(\alpha)$, its eigenvalues are the products of the pairs of distinct eigenvalues of $\alpha$, i.e. of distinct roots of $p(x)$.

If $x=\lambda$ is a root of $p(x)$ then $y=\lambda-\lambda^{-1}$ is a root of $r(y)=y^{2}-d y+2$. If we choose $d=2$ then $r$ has $1+i$ as a root. Thus the roots of $p(x)$ are $\lambda, \bar{\lambda}, \lambda^{-1}, \bar{\lambda}^{-1}$ where $|\lambda|>1$ and $\lambda-\lambda^{-1}=1+i$. The largest positive root of $q(t)$ is $1+\sqrt{2}$, and one 
can easily show $\lambda \bar{\lambda}>1+\sqrt{ } 2$. Thus our example is the companion matrix of $x^{4}-2 x^{3}+$ $2 x+1$.

\section{A possible counterexample}

We present here an isotopy class on $T^{3}$ for which the methods of the preceding sections cannot produce an isentropic fitting. This is possibly a counterexample to the conjecture of Shub mentioned in the introduction.

A handle structure on a connected closed 3-manifold determines a Heegard splitting into the 0 - and 1-handles on the one hand and the 2- and 3-handles on the other. We call the genus of these handlebodies the genus of the handle decomposition. For $T^{3}$ the least genus of a handle decomposition is clearly 3: three 1-handles are needed to generate $H_{1}$ and the standard handle structure has genus 3. We present an isotopy class that has no fitted representative for a genus 3 handle decomposition of least entropy.

Our example was suggested by a remarkable theorem [13]: If a monic integral polynomial is not reciprocal then it has roots whose product has modulus $\geq \theta$ where $\theta$ is the positive root of $p(x)=x^{3}-x-1$. In our setting, this means that a toral automorphism with $p(x)$ as characteristic polynomial has very low entropy and so ought to be difficult to fit isentropically. Indeed,

THEOREM 3. If $\bar{\alpha}: T^{3} \hookleftarrow$ has characteristic polynomial $p(x)$ as above, then any diffeomorphism $f$ homotopic to $\bar{\alpha}$ that is fitted for a genus 3-handle decomposition of $T^{3}$ satisfies $h(f)>h(\bar{\kappa})$.

Proof. Suppose $h(f) \leq h(\bar{\alpha})$. We show that the 1-handles can be ordered and oriented so $A=A_{1}(f)$ is the companion matrix of $p(x)$. It is easy to see then in this case $\Lambda^{2} A$ contradicts lemma 3 .

Let $G=G_{1}(f)$. Lemma 3 implies $G=|A|$ has the same characteristic polynomial as $A$, namely $p(x)$. As $G$ has trace 0 we find $A_{i i}=0$ for all $i$. Also $G$ and $A$ have determinant 1 so there must be an off-diagonal zero entry. Reordering the basis, we may suppose the $A_{21}=0$. We then have $A_{23} A_{31} A_{12}=1$ and by reorienting the 1 -handles we may take these three entries to be +1 . Taking the remaining coefficient of the characteristic polynomial into account, we find that $A_{13}, A_{32}$ are 0 and 1 in some order. Now reorder the basis if needed.

It would be most interesting to know if any isentropic fitting of $\bar{\alpha}$ exists. Maller's thesis should be helpful here [7]. By theorem 2 all sufficiently high iterates of $\bar{\alpha}$ have ISA's. In any case, the preceding theorem and the ergodic counterexamples of [6] explain why we made our conjecture only for sufficiently hyperbolic automorphisms.

This search was partially supported by the National Science Foundation.

\section{REFERENCES}

[1] R. Bowen. One-dimensional hyperbolic sets for flows. J. Diff. Eqns. 12 (1972), 173-179.

[2] R. Bowen. Entropy for group endomorphisms and homogeneous spaces. Trans. Amer. Math. Soc. 153 (1971), 377-397. 
[3] M. Denker, C. Grillenberger \& K. Sigmund. Ergodic Theory on Compact Spaces. Lecture Notes in Math. No. 527. Springer: Berlin, 1976.

[4] J. Franks. Anosov diffeomorphisms. Global Analysis XIV (Proc. Symp. Pure Math. 1968), pp. 61-93. Amer. Math. Soc.: Princeton, 1968.

[5] J. Franks. Homology and Dynamical Systems. Notes from conf. at Emory University 1980.

[6] D. Fried. Efficiency vs. hyperbolicity on tori. Global Theory of Dynamic Systems. Lecture Notes in Maths. No. 819. Springer: Berlin, 1980.

[7] M. Maller. Fitted diffeomorphisms on non-simply connected manifolds. Topology 19 (1980), 395-410.

[8] A. Manning. There are no new Anosov diffeomorphisms on tori. Amer. J. Math.96(1974), 422-429.

[9] S. E. Newhouse. On codimension one Anosov diffeomorphisms. Amer. J. Math. 92 (1970), 761-770.

[10] M. Shub. Dynamical systems, filtrations and entropy. Bull. Amer. Math. Soc. 80 (1974), $27-41$.

[11] M. Shub \& D. Sullivan. Homology theory and dynamical systems. Topology 14 (1975), 109-132.

[12] Ja. G. Sinai. Construction of Markov partitionings. Func. Anal. Appl. 2 (1968), 39-52.

[13] C. J. Smyth. On the product of the conjugates outside the unit circle of an algebraic integer. Bull. Lond. Math. Soc. 3 (1971), 169-175. 